\title{
Promovendo saúde bucal e entretenimento a crianças de comunidades carentes através da extensão universitária
}

\author{
Promoting oral health and entertainment for children from underprivileged communities through \\ university extension
}

Promover la salud bucal y el entretenimiento para niños de comunidades desfavorecidas a través de la extensión universitária

Lorena Layanne Pereira Custódio

ORCID: https://orcid.org/0000-0002-6379-5993 Universidade Federal de Campina Grande, Brasil E-mail: lorenalayanne888@gmail.com

Gabrielli Bezerra Sales

ORCID: https://orcid.org/0000-0003-1842-9844 Universidade Federal de Campina Grande, Brasil E-mail: gabrielli.b.sales@gmail.com

Myllena Silva Queiroz

ORCID: https://orcid.org/0000-0003-0757-1992 Universidade Federal de Campina Grande, Brasil E-mail: Myllenaqueiroz959@gmail.com

Rafaela Oliveira Santos ORCID: https://orcid.org/0000-0002-3072-8874 Universidade Federal de Campina Grande, Brasil E-mail: rafaela_ob@hotmail.com

Gymenna Maria Tenório Guênes ORCID: https://orcid.org/0000-0002-5447-0193 Universidade Federal de CampinaGrande, Brasil E-mail: gymennat@yahoo.com.br

Elizandra Silva da Penha

ORCID: https://orcid.org/0000-0001-6264-5232 Universidade Federal de Campina Grande, Brasil E-mail: elizandrapenha@hotmail.com

Luanna Abílio Diniz Melquíades de Medeiros ORCID: https://orcid.org/0000-0002-1630-3968 Universidade Federal de Campina Grande, Brasil E-mail: luannaabiliod@gmail.com

Camila Helena Machado da Costa Figueiredo ORCID: https://orcid.org/0000-0002-1340-4042 Universidade Federal de Campina Grande, Brasil E-mail: camila_helena_@hotmail.com

\begin{abstract}
Resumo
A extensão universitária, no âmbito odontológico, fundamenta-se na prestação de serviços à saúde bucal da população, através de diversos e significativos programas. Este trabalho objetiva relatar as experiências e atividades vivenciadas pelos alunos de Odontologia da Universidade Federal de Campina Grande integrantes do Programa de Extensão (PROBEX): "Calouros Humanos: Promovendo Saúde Bucal na Pastoral da Criança", pertencentes ao eixo direcionado às ações de promoção de saúde bucal com crianças através de práticas educativas em comunidades do município de Patos, Paraíba (PB). Participaram das ações crianças de 0 a 12 anos de idade, assim como seus pais e responsáveis que frequentavam a pastoral da criança em suas comunidades. O Eixo das Crianças contou com a participação de 8 integrantes, sendo: 1 professora coordenadora, 1 aluno bolsista e 6 alunos voluntários, os quais reuniam-se a cada semana para agendar e fazer o planejamento das ações com o intuito de disseminar conhecimento a respeito de saúde bucal. O programa proporciona uma disseminação de informações seguras, claras e precisas, que se adequam às crianças, de forma lúdica e de fácil compreensão, possibilitando um maior aprendizado. Além disso, as ações favorecem o processo de ensino e desenvolvimento do aluno extensionista, uma vez que esse convívio com a comunidade permite a interação social deste com diversas pessoas permitindo a construção de um profissional capacitado na prevenção e promoção de saúde.
\end{abstract}

Palavras-chave: Odontologia; Saúde bucal; Extensão; Criança. 


\begin{abstract}
The university extension, in the dental field, is based on the provision of services to the oral health of the population, through several and significant programs. This work aims to report the experiences and activities experienced by Dentistry students at the Federal University of Campina Grande, members of the Extension Program (PROBEX): "Human Freshmen: Promoting Oral Health in Pastoral da Criança", belonging to the axis directed to actions to promote oral health with children through educational practices in communities in the city of Patos, Paraíba (PB). Children from 0 to 12 years of age participated in the actions, as well as their parents and guardians who attended the Pastoral da Criança in their communities. The Children's Axis had the participation of 8 members, namely: 1 coordinating teacher, 1 scholarship student and 6 volunteer students, who met every week to schedule and plan actions in order to disseminate knowledge about it of oral health. The program provides safe, clear and accurate information dissemination, which is suitable for children, in a playful and easy to understand way, enabling greater learning. In addition, the actions favor the process of teaching and developing the extension student, since this contact with the community allows social interaction with different people, allowing the construction of a professional trained in prevention and health promotion.
\end{abstract}

Keywords: Dentistry; Oral health; Extension; Child.

\title{
Resumen
}

La extensión universitaria, en el ámbito odontológico, es fundamental en la prestación de servicios a la salud bucal de la población, a través de varios y contrastados programas. Este trabajo tiene como objetivo relatar las experiencias y actividades vividas por los estudiantes de Odontología de la Universidad Federal de Campina Grande, miembros del Programa de Extensión (PROBEX): "Humanos de primer año: Promoción de la salud bucal en la Pastoral da Criança", perteneciente al eje dirigido a la salud. acciones de promoción con niños a través de prácticas educativas en comunidades de la ciudad de Patos, Paraíba (PB). En las acciones participaron niños de 0 a 12 años, así como sus padres y tutores que asistieron a la pastoral del niño en sus comunidades. El Eje Infantil contó con la participación de 8 integrantes, a saber: 1 docente coordinador, 1 estudiante becado y 6 estudiantes voluntarios, quienes se reunieron semanalmente para programar y planificar acciones con el fin de difundir conocimientos en el respeto a la salud bucal. El programa ofrece una difusión clara y precisa de información de seguridad, adecuada para los niños, de una manera lúdica y comprensiva, lo que permite un mayor aprendizaje. Además, las acciones favorecen el proceso de enseñanza y desarrollo del alumno extensionista, ya que este contacto con la comunidad permite una interacción social entre el alumno y varias personas, permitiendo la construcción de un profesional capacitado en prevención y promoción de la salud.

Palabras clave: Odontología; Salud bucal; Extensión; Niño.

\section{Introdução}

Com a promulgação das diretrizes curriculares nacionais no Brasil, os perfis curriculares dos cursos de Odontologia foram alterados requerendo do acadêmico e futuro profissional uma formação humanizada, generalista, crítica e reflexiva, agindo em todos os níveis de atenção à saúde (Moura et al., 2012). Elaborar competências para o desenvolvimento de programas embasados em treinamentos destinados ao atendimento primário futuro é atribuição da Universidade, incluindo o aluno na prática hábil em saúde com a comunidade (Conto, 2013).

A extensão universitária associa o ensino e a pesquisa e possibilita a relação transformadora entre a universidade e a sociedade, compreendendo as demandas impostas por cada comunidade. Seu desenvolvimento pode ocorrer por diferentes modalidades, como: projetos, programas, cursos e oficinas, eventos e prestação de serviços. Através dela, acontece o processo educativo, cultural e científico, onde é viável estabelecer o diálogo e socializar o conhecimento entre o saber popular e científico. Essa interação é uma estratégia significativa para compreensão e possível transformação da realidade em saúde, modificando a metodologia tradicional de formação do profissional (Fadel, Bordin, Kuhn \& Martins, 2013; Ribeiro, Araújo \& Silva, 2017; Silva, 2020; Mélo et al., 2021).

Na Odontologia, a extensão universitária fundamenta-se na prestação de serviços à saúde bucal da população, através de diversos e significativos programas, porém, não apenas de caráter assistencial, mas com um papel interligado à prática acadêmica, ao ensino e à pesquisa aplicada. Nesta, geralmente, há um número elevado de discentes e docentes envolvidos no desenvolvimento de ações, buscando não substituir, mas contribuir nesse âmbito de políticas sociais (Conto, 2013). Por meio 
dessa interação entre a sociedade e o meio acadêmico ocorre uma adaptação às linguagens e vivências, produzindo diálogos relevantes e necessários (Santos et al., 2020).

Nesse contexto, a promoção em saúde atua como um processo de capacitação da comunidade buscando melhorias em sua qualidade de vida e saúde, onde a mesma participará ativamente no controle de tais aspectos no seu cotidiano. Dentre as estratégias desenvolvidas a mobilização social e a parceria estabelecida com a população (Macedo, Santos Pacheco, Degli Esposti, Carvalho \& Pacheco Filho, 2017; Cardoso et al., 2020).

Segundo Taglietta et al. (2011), as crianças em idade escolar possuem uma maior facilidade em adquirir conhecimentos e desenvolver hábitos de educação em saúde, hábitos alimentares e incorporar os cuidados à saúde bucal de forma correta e eficiente, sendo estes comportamentos firmados e resistentes a modificações futuras. A educação em saúde é um aspecto transformador na realidade social, direcionada por ações voluntárias, individuais ou coletivas, que prezam pelo autocuidado em saúde e pelo bem da comunidade, configurando-se como uma promoção em saúde valorosa (Júnior, Leal, Ferreira \& Gonçalves, 2019; Meneses, Barbosa, Wanderley \& Bandini, 2021).

Considerando o exposto, este relato objetiva apresentar as experiências e atividades vivenciadas pelos alunos extensionistas integrantes do Programa Calouros Humanos: Promovendo Saúde Bucal na Pastoral da criança, pertencentes ao eixo direcionado às ações de transmissão de conhecimentos e práticas de saúde bucal desenvolvidas com crianças de comunidades do município de Patos, Paraíba. Sendo este Programa uma extensão universitária desenvolvida por acadêmicos do curso de Odontologia da Universidade Federal de Campina Grande (UFCG), Campus Patos, PB.

\section{Metodologia}

O presente trabalho trata-se de um estudo descritivo, qualitativo, do tipo relato de experiência, referente à experiência de um sub eixo do Programa de Extensão (PROBEX): "Calouros Humanos - Promovendo Saúde Bucal na Pastoral da Criança”, enfatizando a promoção de saúde bucal às crianças através de práticas educativas. Com finalidade de descrever as experiências, possibilitando aprimorar a familiaridade com campo de estudo, através da coleta e análise de dados obtidos diretamente do seu ambiente natural ou da realidade presenciada (Estrela, 2018; Pereira et al., 2018).

A experiência foi realizada com crianças que continham de 0 a 12 anos de idade, assim como seus pais e responsáveis que frequentavam a pastoral da criança de comunidades carentes localizadas no município de Patos, no estado da Paraíba.

O Eixo das Crianças contou com a participação de 8 integrantes, sendo: 1 professora coordenadora, 1 aluno bolsista e 6 alunos voluntários, os quais reuniam-se a cada semana para agendar e fazer o planejamento das ações. Estes realizavam ações mensais, geralmente nas sextas-feiras, nas paróquias das igrejas da cidade. As ações foram realizadas na vigência do ano de 2019, compreendendo os meses de abril a dezembro.

Ao todo foram realizadas 8 ações com o intuito de disseminar conhecimento a respeito de saúde bucal, com tentativa de abordar principalmente aquelas comunidades mais carentes. Antes de cada ação, os extensionistas buscavam quais os principais problemas enfrentados pela população, além das principais comodidades relatadas pelo responsável da pastoral. Sendo assim, as ações eram baseadas nessas informações, as quais eram levadas em consideração para que esses pontos fossem planejados e abordados durante as palestras e dinâmicas apresentadas.

O foco principal foi passar informações através de rodas de conversa, palestras jogos, músicas e teatro, mostrar a importância da saúde bucal e como pode influenciar na saúde sistêmica, temas como alimentação e higiene oral utilizando macro modelos para intensificar o processo de aprendizagem, com uma melhor visualização da cavidade oral. Além disso, eram confeccionados banners com imagens sobre os diversos temas abordados e o que poderia ser feito em cada uma das situações. 
Desenhos ilustrativos para pintura, vídeos, paródias, dinâmicas e apresentações lúdicas voltados para Odontologia eram preparados e sempre abordados ao final de cada ação como forma fortalecer todos os conhecimentos que tinham sido abordados até então. Após todas as atividades era servido lanches e entregue brindes a todos os indivíduos que haviam participado das ações.

Além das atividades com as crianças, os pais e responsáveis presentes recebiam informações acerca da sua saúde bucal bem como dos seus filhos. Temas como traumatismo, câncer oral, automedicação, saúde da gestante também eram abordadas nas comunidades, além de apresentar a estes os atendimentos e serviços disponibilizados pela clínica escola da UFCG a fim de trazer benefícios para a comunidade através dos tratamentos preventivos e curativos prestados.

\section{Sobre a Pastoral da Criança}

A Pastoral da Criança (https://www.pastoraldacrianca.org.br), recuperado em 01 de junho, 2021) é uma entidade sem fins lucrativos, a qual atua por meio de uma organização da comunidade, através de líderes, os quais são preparados para orientarem e acompanharem as famílias. Os líderes são voluntários, e por fazerem parte da comunidade, dão suporte às famílias e promovem ações de saúde e educação, para as crianças e seus responsáveis.

O principal objetivo da Pastoral da Criança é a diminuição de mortes materno-infantis, além de garantir ambientes favoráveis para que os mesmos se desenvolvam com segurança e estabilidade.

\section{Figura 1.}

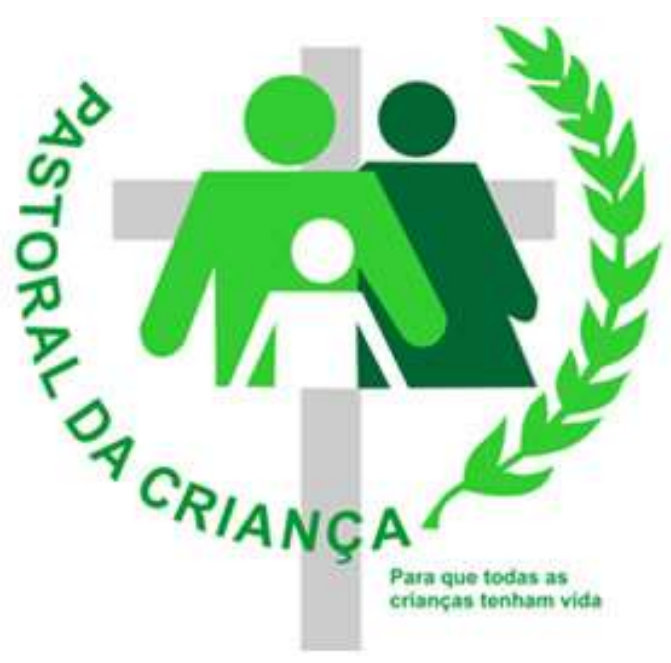

Fonte: Pastoral da criança.

\section{Sobre o Programa de Extensão Universitária}

Extensão universitária é estender olhares sobre as comunidades, é compreender o lado biopsicossocial do indivíduo e praticar a humanização. Por essa razão, e com o intuito de unir anseios e expectativas dos calouros do curso de odontologia juntamente com os conhecimentos e experiências dos veteranos surgiu o programa Calouros Humanos - eixo das crianças - da Universidade Federal de Campina Grande, campus de Patos - Paraíba, com ênfase na relação teoria-prática, na perspectiva de uma relação dialógica entre universidade e sociedade, como oportunidade de troca de saberes dentro da concepção em saúde bucal.

O Programa Calouros Humanos tem por objetivo o desenvolvimento e aplicação de atividades e conteúdos relacionados à saúde bucal dentro do âmbito da Pastoral da Criança, na cidade de Patos, PB, reunindo os discentes do primeiro 
período de Odontologia da Universidade Federal de Campina Grande, para a execução dessas ações, tornando essa vivência reconhecida desde o início da vida acadêmica.

\section{Relato de Experiência}

Inicialmente, o Programa Calouros Humanos foi apresentado à turma dos alunos do primeiro período de Odontologia, para que conhecessem o programa e pudessem se organizar para participar de determinadas ações. Logo após, foi feita uma reunião com os extensionistas para debater sobre os temas mais pertinentes a serem desenvolvidos, além da reforma e confecção de novos objetos e brinquedos lúdicos que eram utilizados para as atividades nas comunidades.

Quando os alunos do primeiro período de Odontologia iriam participar de forma ativa nas ações, a turma era dividida em grupos e a dinâmica das ações era organizada previamente, sendo desenvolvidas pelos próprios calouros, para uma interação mais ativa e livre com a comunidade. Os encontros nas comunidades eram realizados de forma que os extensionistas tinham um primeiro contato com a comunidade, realizando uma primeira ação e, posteriormente, os alunos do primeiro período realizavam a ação seguinte, com o auxílio dos extensionistas.

A primeira ação aconteceu no Bairro do Bivar Olinto, Patos -PB, na comunidade Nossa Senhora das Neves, contando com a participação de aproximadamente 50 crianças. Nesta ação, foram desenvolvidas atividades lúdicas, como: pintura educativa em papel, pintura facial, teatro educativo sobre educação em saúde bucal e importância de hábitos saudáveis, brincadeira do "dente vivo e dente morto", foi feita a orientação de higiene bucal com o macromodelo de boca e escova, com posterior participação das crianças para elas mostrarem o que foi aprendido. Ao final de cada ação era servido um lanche e distribuído brindes para todas as crianças.

A segunda ação foi realizada no bairro Santo Antônio, tendo a participação dos extensionistas e calouros e a presença de cerca de 50 crianças. Nessa ação, os calouros puderam desenvolver as atividades com autonomia, realizando várias dinâmicas com as crianças. Foram desenvolvidas cantigas de roda, pinturas faciais, jogo da memória educativo, dança, orientação de higiene bucal e etc. Por fim, as crianças receberam kits de escovação, onde os calouros puderam realizar a escovação supervisionada.

Na Comunidade São Francisco, no bairro Jardim Redenção, foi realizada a terceira ação, tendo aproximadamente 140 crianças. Nessa ação foi realizada uma gincana com diversas provas, como: perguntas e respostas, estoure o balão, "acerte a fruta na boca do dentão", dinâmica da evolução da cárie dentre outras. Além disso, as crianças fizeram pintura educativa em papel e receberam pintura facial. Ao final, orientamos as crianças sobre a higiene bucal correta com o macromodelo de boca e escova.

A quarta ação foi realizada na Comunidade São Paulo, com a presença de cerca de 50 crianças. Nesta comunidade, foram realizados jogos de memória educativos, brincadeira do vivo ou morto e da "batata quente", sendo realizada uma pergunta sobre higiene bucal na criança onde a música parasse, pintura educativa em papel, cantigas de roda, entre outras brincadeiras.

A ação seguinte foi na Comunidade Vila Mariana, no Santuário Cruz da Menina, tendo a participação dos calouros de Odontologia, contando com a presença de 65 crianças. Neste dia, os calouros desenvolveram atividades com as crianças, como: jogos com bola, pintura educativa no papel, pintura facial, brincadeiras de roda com cantigas educativas, além de terem feito a demonstração de como se faz uma higienização bucal correta. Em seguida, os calouros se paramentaram para realizar a escovação supervisionada com as crianças, que receberam kits de escovação.

Foi realizada mais uma ação, a qual foi na Comunidade Itatiunga, contando com a participação de cerca de 100 crianças, os extensionistas e calouros. No início, a equipe da Pastoral da Criança fez a abertura do momento com orações, já que a Pastoral foi fundada pela igreja juntamente com a comunidade. A ação foi dedicada ao mês das crianças e foi 
desenvolvida pelos calouros. Foi realizada pintura facial, brincadeiras de roda, pintura educativa em papel, jogos, gincanas, dentre outras atividades. Após isso, as crianças receberam os kits de escovação e os calouros puderam realizar a escovação supervisionada.

A ação realizada na Comunidade Dom Bosco contou com a participação de 50 crianças. Foi desenvolvida atividade teatral educativa, com ênfase nos hábitos de alimentação saudável, brincadeira do dente bom e dente ruim, rodas de música, pintura facial e pintura educativa em papel.

A última ação aconteceu na Comunidade São Sebastião, no Bairro São Sebastião, contando com a participação de cerca de 35 crianças. Nesta ação, desenvolvemos algumas atividades, como: brincadeira do dente bom e dente ruim (evidenciando quais alimentos são bons e quais são ruins para os dentes), pintura facial, jogos da memória, brincadeira do vivo ou morto, pintura educativa em papel, entre outras. Por fim, foi feita a orientação de higiene bucal às crianças de forma lúdica.

\section{Discussão}

Desde os primórdios, o lúdico sempre esteve presente na vida dos seres humanos, especialmente das crianças, pois é nessa fase da vida que os jogos e brincadeiras são as atividades mais importantes e atrativas do dia, tornando-se cruciais para o desenvolvimento intelectual e motor desses indivíduos (Santos, Costa \& Martins, 2015). Segundo Santos et al. (2020), o comportamento de cada criança sofre influência direta de acordo com o ambiente que vivem, assim, para que o programa seja eficaz, suas ações devem ser planejadas e realizadas adaptando-se à realidade de cada comunidade.

O uso adequado de atividades lúdicas como jogos educativos, macromodelos, músicas e apresentações assumem um papel essencial na promoção e educação em saúde bucal infantil, pois estimulam a adoção dos hábitos, atraem a atenção e incentivam as crianças a seguirem as informações repassadas durante as dinâmicas. Os profissionais podem utilizar diversos recursos, de acordo com suas afinidades e a necessidade de cada local, de forma que o conhecimento seja transmitido de maneira simples e satisfatória para todos os participantes da ação, na tentativa de melhorar a qualidade de vida de todos os envolvidos (Cota \& Costa, 2017).

De acordo com do Nascimento et al. (2020), as ferramentas lúdicas realizadas de forma simples possibilitam a disseminação da informação inclusiva e dinâmica, sendo bastante eficaz para adequar-se e despertar o interesse do público envolvido. Assim, tendo em vista a importância dessas atividades lúdicas, as ações do programa Calouros Humanos foram planejadas e pensadas de acordo com o público abordado com o intuito de tornar a experiência engrandecedora de modo a conseguir atrair atenção das crianças. Dessa forma, é fundamental que os extensionistas estejam aptos para tais ações, contribuindo para um programa de excelência.

A realização dessas ações educativas voltadas à prevenção, abordando temas como orientação de higiene oral, uso do flúor e aconselhamento dietético são extremamente importantes na preservação da saúde bucal, repercutem de maneira positiva tanto individualmente como de forma coletiva, além de ser de baixo custo quando comparado ao tratamento curativo, como a realização de restaurações e exodontias (Santos, Casotti, Guimarães \& Freitas Uemura, 2015). Além das crianças, destaca-se também a necessidade de participação dos pais e responsáveis durante as ações, tendo em vista que o cuidado com a saúde bucal dessa faixa etária geralmente é realizado por um adulto (Araújo, 2015).

Assim, a experiência vivenciada propiciou aos acadêmicos de Odontologia um novo olhar para a educação em saúde bucal. Ampliando o conceito de humanização do processo educativo em saúde, agregando conhecimentos e benefícios oferecidos, através da atuação em comunidades necessitadas. Além da melhor compreensão dos diversos cenários de atuação, tendo em vista que serão futuros profissionais da saúde. 


\section{Considerações Finais}

A conscientização e a educação em saúde bucal para essas comunidades menos assistidas do município de Patos, faz com que haja a difusão do conhecimento odontológico, além disso, desmistifica possíveis receios enfrentados pelas crianças. O aprendizado infantil deve ser atrativo e interativo, fazendo com que eles possam se interessar e fazer desses ensinamentos uma rotina diária em suas casas.

Por fim, podemos afirmar que as atividades educativas lúdicas possibilitam um maior aprendizado para as crianças, como também favorecem o processo de ensino e desenvolvimento do aluno extensionista, uma vez que esse convívio com a comunidade permite a interação social do aluno com diferentes pessoas, diversas faixas-etárias e condições socioeconômicas e culturais diversas, permitindo a construção de um profissional capacitado na prevenção em saúde, assim como mais sensível no convívio com a população em geral.

Para isso, é importante que haja a ampliação de estudos e pesquisas voltadas para essa área, uma vez que, através do conhecimento dessas práticas, estudantes de diversas localidades e cursos podem ser incentivados a desenvolverem atividades voluntárias e buscarem o bem-estar das comunidades a que pertencem, permitindo um olhar mais crítico e humanizado, além dos conteúdos técnicos e do interior da universidade.

\section{Referências}

Araújo, P. C. (2015). Avaliação comparativa entre as escolas municipais de ensino básico que receberam e as que não receberam o programa de promoção em saúde bucal da Faculdade de Odontologia de Araçatuba. Universidade Estadual Paulista Júlio de Mesquita Filho, 01-93.

Cardoso, S. D. A. M., de Sousa, M. R. R., Uchôa, I. S., Freitas, F. A. P. S., Nascimento, S. T. T., \& Magalhães, M. D. A. V. (2020). Atendimento Odontológico na Zona Rural do Piauí: A extensão colaborando com a experiência acadêmica. Research, Society and Development, 9(11), e4859119897e4859119897. https://doi.org/10.33448/rsd-v9i6.3677

Conto, F de. (2013). A extensão universitária na faculdade de odontologia. Revista Em Extensão, 12(2), 100-108. https://doi.org/10.14393/REEv12n22013_rel01

Cota, A. L. S., \& Costa, B. J. D. A. (2017). Atividades lúdicas como estratégia para a promoção da saúde bucal infantil. Saúde e pesquisa, 365-371. https://doi.org/10.17765/2176-9206.2017v10n2p365-371

Estrela, C. (2018). Metodologia Científica: Ciência, Ensino, Pesquisa. Editora Artes Médicas.

Fadel, C. B., Bordin, D., Kuhn, E., \& Martins, L. D. (2013). O impacto da extensão universitária sobre a formação acadêmica em Odontologia. InterfaceComunicação, Saúde, Educação, 17(47), 937-946. https://doi.org/10.1590/1807-57622013.3811

Júnior, H. D. S. C., Leal, R. L., Ferreira, C., \& Gonçalves, B. A. D. O. C. (2019). Saúde bucal para a educação infantil: uma análise entre professores do município de porto nacional. Revista Científica do ITPAC, 12(2), 10-17.

Macedo, L. R., Santos Pacheco, K. T., Degli Esposti, C. D., de Carvalho, R. B., \& Pacheco Filho, A. C. (2017). Promoção de saúde bucal para pré-escolares: relato de experiência. Revista Ciência em Extensão, 13(4), 128-139.

Mélo, C. B., Farias, G. D., Nunes, V. R. R., de Andrade, T. S. A. B., \& Dalle Piagge, C. S. L. (2021). A extensão universitária no Brasil e seus desafios durante a pandemia da COVID-19. Research, Society and Development, 10(3), e1210312991-e1210312991. https://doi.org/10.33448/rsd-v10i3.12991

Meneses, P. V. S., Barbosa, É. P., Wanderley, F. A. C., \& Bandini, C. S. M. (2021). Atividades lúdicas para promoção de saúde bucal em escolares: revisão de literatura. Revista Eletrônica Acervo Saúde, 13(2), e5726-e5726. https://doi.org/10.25248/reas.e5726.2021

Moura, L. D. F. A. D. D., Piauilino, R. J. B., Araújo, Í. F., Moura, M. S. D., Lima, C. C. B., Evangelista, L. D. M., \& Lima, M. D. D. M. D. (2012). Impacto de um projeto de extensão universitária na formação profissional de egressos de uma universidade pública. Revista de Odontologia da UNESP, 41(5), 348-352.

Nascimento, L. S. do, Lima, F. O., Goes, V. N., Nogueira, P. L., Silva, Q. P., Macena, M. C. B., Rodrigues, R. Q. F., \& Fonseca, F. R. A. (2020). Saúde oral de forma lúdica através de atividades de extensão universitária em uma Organização Não Governamental (ONG). Research, Society and Development, 9(6), 112. https://doi.org/10.33448/rsd-v9i6.3677

Pereira A. S. et al. (2018). Metodologia da pesquisa científica. UFSM.

Ribeiro, M. R. F., Araújo, V. M. P. de, \& Silva, E. A. (2017). A contribuição da extensão universitária na formação acadêmica: desafios e perspectivas. Revista Conexão UEPG, 13(1), 52-65. https://doi.org/10.5212/Rev.Conexao.v.13.i1.0004

Santos, C. C. S. da, Costa, L. F. da, \& Martins, E. (2015). A prática educativa lúdica: uma ferramenta facilitadora na aprendizagem na educação infantil. Revista Eletrônica do Curso de Pedagogia das Faculdades OPET, 74-89. 
Research, Society and Development, v. 10, n. 13, e488101321292, 2021

(CC BY 4.0) | ISSN 2525-3409 | DOI: http://dx.doi.org/10.33448/rsd-v10i13.21292

Santos, L. G. P., Rodrigues, D. C., Mariz, W. S., Torres, J. L. M., Pinheiro, N. S. D. S. L., Macena, M. C. B., Rodrigues, R. Q. F., \& Fonseca, F. R. A. (2020). Manejo do comportamento infantil e saúde bucal na sala de espera: um relato de experiência. Research, Society and Development, 9(7), 1-12. https://doi.org/10.33448/rsd-v9i7.5069

Santos, M. O., Casotti, C. A., Guimarães, A. P. D. de, \& Freitas Uemura, T de. (2015). Conhecimento e percepção sobre saúde bucal de professores e préescolares de um município baiano. Revista da Faculdade de Odontologia-UPF, 20(2), 172-178. https://doi.org/10.5335/rfo.v20i2.4689

Santos, R. O., Sales, G. B., Custódio, L. L. P., Queiroz, M. S., Guênes, G. M. T., da Penha, E. S., \& da Costa Figueiredo, C. H. M. (2020). O uso dos meios de comunicação como forma de promoção de saúde bucal: experiência de acadêmicos de Odontologia. Research, Society and Development, 9(8), e596986275e596986275. http://dx.doi.org/10.33448/rsd-v9i8.6275

Silva, A. R. J da. (2020). Extensão universitária na odontologia como uma experiência em saúde. Revista Saúde, 16(1), 1761-1765. https://doi.org/10.22481/rsc.v16i1.4697

Taglietta, M. F. A., Bittar, T. O., Brandão, G. A. M., Lima Vazquez, F. d, Paranhos, L. R., \& Pereira, A. C. (2011). Impacto de um programa de promoção de saúde escolar sobre a redução da prevalência da cárie em crianças pré-escolares de Piracicaba-SP. Revista da Faculdade de Odontologia-UPF, 16(1), 13-17. https://doi.org/10.5335/rfo.v16i1.2104 